Nanoscale roughness effect on Maxwell-like boundary conditions

\title{
Nanoscale roughness effect on Maxwell-like boundary conditions for the Boltzmann equation
}

S. Brull, ${ }^{1, a)}$ P. Charrier, ${ }^{1, b)}$ and L. Mieussens ${ }^{1, c)}$

Univ. Bordeaux, CNRS, Bordeaux INP, IMB, UMR 5251, F-33400 Talence, France.

(Dated: 18 June 2016)

It is well known that the roughness of the wall has an effect on microscale gas flows. This effect can be shown for large Knudsen numbers by using a numerical solution of the Boltzmann equation. However, when the wall is rough at a nanometric scale, it is necessary to use a very small mesh size which is much too expansive. An alternative approach is to incorporate the roughness effect in the scattering kernel of the boundary condition, such as the Maxwell-like kernel introduced by the authors in a previous paper. Here, we explain how this boundary condition can be implemented in a Discrete Velocity approximation of the Boltzmann equation. Moreover, the influence of the roughness is shown by computing the structure scattering pattern of mono-energetic beams of the incident gas molecules. The effect of the angle of incidence of these molecules, of their mass, and of the morphology of the wall is investigated and discussed in a simplified two-dimensional configuration. The effect of the azimuthal angle of the incident beams is shown for a three-dimensional configuration. Finally, the case of non-elastic scattering is considered. All these results suggest that our approach is a promising way to incorporate enough physics of gas-surface interaction, at a reasonable computing cost, to improve kinetic simulations of micro and nano-flows.

PACS numbers: 51.10, 34.35, 02.70

Keywords: gas-surface interaction, kinetic theory

\footnotetext{
a)Electronic mail: Stephane.Brull@math.u-bordeaux.fr

b)Electronic mail: Pierre.Charrier@math.u-bordeaux.fr

c)Electronic mail: Luc.Mieussens@math.u-bordeaux.fr
} 
Nanoscale roughness effect on Maxwell-like boundary conditions

\section{INTRODUCTION}

In some applications we have to consider flows at high Knudsen number on domains bounded by walls. Examples of such situations are external flows at high altitude around re-entry bodies or internal flows in micro or nano devices (MEMS or NEMS) and in sub-micrometer porous media. In such configurations the flow is well described by a kinetic equation (the Boltzmann equation or the BGK model), together with boundary conditions imposed on the wall. With more and more powerful computers such models are now currently used for numerical simulations. A boundary condition for the Boltzmann equation is a way to take into account, at the kinetic scale, the complex gas-surface interaction which takes place at a very small scale (typically on less than one nanometer). The most used boundary conditions are the Maxwell and the Cercignani-Lampis conditions ( $\mathrm{see}^{1}$ for a detailed review on boundary conditions and ${ }^{3}$ for recent works). However none of them take into account some small or medium scale roughness of the wall, though it is well-known that such roughness may notably affect the flow near the wall. As a result, those standard boundary conditions may turn out to be unable to describe with enough accuracy the transport properties of the flow near the surface.

To overcome this difficulty for the Maxwell condition, a mathematical framework, recently introduced by the authors ${ }^{4}$, allows to derive boundary conditions for a periodic rough wall by using a rigorous upscaling method. In the simplest case, corresponding to a flat wall, this condition looks like a Maxwell condition but with accommodation coefficients that depend on the velocity of the impinging molecules

and on the temperature and morphology of the wall (see another recent approach ${ }^{2}$ that also leads to variable accommodation coefficients). At the small scale the gassurface interactions are assumed to be governed by a potential energy surface (PES) which was not specified $\mathrm{in}^{4}$.

In the present paper we propose a simple but physically reasonable PES and a way to incorporate it in this mathematical framework, in order to get an improved boundary condition for the Boltzmann equation easy to implement for numerical 
Nanoscale roughness effect on Maxwell-like boundary conditions

simulations with a discrete velocity method (DVM). Many contributions on the gas-surface interaction have been published in past years either using very simple models, like the "hard cube" model ${ }^{5}$ or the washboard model ${ }^{6}$ or much more elaborate ones ${ }^{7} 8$. Here we suggest to use an intermediate model, simple enough to be tractable, but precise enough to include at the kinetic scale the effect of van der Waals forces and of roughness at atomic scales. The PES is defined by a lattice of Lennard-Jones atoms. In an auxiliary problem, for given incident velocities of impinging molecules, we compute the trajectories of a nanoscopic sample of gas atoms in a periodic cell of the surface layer, in which gas molecules interact with surface atoms by the simplified PES. From those data computed once and for all, we get an approximation of the reflection kernel of the new boundary condition suitable for DVM numerical simulations of the Boltzmann equation. At the same time we obtain the scattering pattern of mono-energetic beams of incident gas atoms by the wall and we can visualize and analyze the ability of our approach to incorporate at the kinetic level information from the atomic scale.

The paper is organized as follows: Section II reminds the modified Maxwell-like boundary condition ${ }^{4}(\mathrm{MLBC})$ and its derivation for elastic scattering and non-elastic scattering as well. In section III we describe how to implement this MLBC in a DVM context. Section IV is devoted to the numerical computation of scattering patterns to analyze the effect of nanoscale roughness. The impact of the key parameters (incident angle and energy of the impinging gas atoms, parameters of the LennardJones potentials) on the so-called structure scattering pattern (elastic scattering) is shown (for 2D and 3D configurations) and some indications are given for non-elastic scattering. The paper closes with some comments and conclusions in section $\mathrm{V}$. 
Nanoscale roughness effect on Maxwell-like boundary conditions

\section{NEW BOUNDARY CONDITIONS FOR THE BOLTZMANN EQUATION}

\section{A. A nanoscale gas-surface interaction model}

We start from a nanoscale model describing the gas-surface interaction which generalizes to rough walls the model introduced $\mathrm{in}^{9}$ and used $\mathrm{in}^{10}$. In this model the interaction between the wall and the single atom gas molecules through van der Waals forces are taken into account in a thin surface layer (with thickness $L$ typically smaller than one nanometer). In all the following (except at the end of section IV A), for the sake of simplicity, we assume that the atoms move in a 2D half-plane and we consider the following configuration: the solid occupies the half-space $z>L$, the gas phase is constituted by the gas atoms in the half-space $z<0$, outside of the range of the surface forces, and we consider separately the surface layer $0<z<L$, where the gas atoms move within the range of the surface potential. The gas flow in this surface layer is modeled by the Boltzmann equation (or a BGK-type model equation), with a Vlasov term to take into account the part of the interaction that depends on the frozen position of the atoms of the solid wall (the long range interactions), and a collision term between gas atoms and phonons to take into account the thermal fluctuations of the atoms of the solid (short range interactions) ( $\operatorname{see}^{9}$ for a physical justification of this approach). We consider (as in ${ }^{4}$ ) the following general configuration for the wall: the interaction potential $\mathcal{V}(x, z)$ is such that

$$
\mathcal{V}(x, z)=\mathcal{V}_{\#}\left(\frac{x}{L_{*}}, z\right)
$$

where $L_{*}=\frac{L}{\lambda_{*}}$ and $\lambda_{*}$ is a positive constant that characterizes the roughness of the wall, and $\mathcal{V}_{\#}(y, z)$ is a periodic function of the nanoscopic variable $y$ with period 1. This nanoscopic variable $y$ allows us to describe how a gas atom impinging the surface layer at a microscopic coordinate $x$ sees the nanoscopic roughness of the wall. Moreover we assume that there exists a 1-periodic function $z=\zeta_{\infty}(y)$ with 
Nanoscale roughness effect on Maxwell-like boundary conditions

$0 \leq \zeta_{\infty}(y)<L$ such that

$$
\begin{aligned}
\lim _{z<\zeta_{\infty}(y), z \rightarrow \zeta_{\infty}(y)} \mathcal{V}_{\#}(y, z) & =+\infty \\
\mathcal{V}_{\#}(y, z) & =\mathcal{V}_{m}, \text { for } z \leq 0
\end{aligned}
$$

Condition (2) is a mathematical way to ensure that no gas atom can penetrate the wall, i.e. that it is a non porous wall and condition (3) means that the potential is constant outside the surface layer (see Figure 1). With these assumptions, the flow

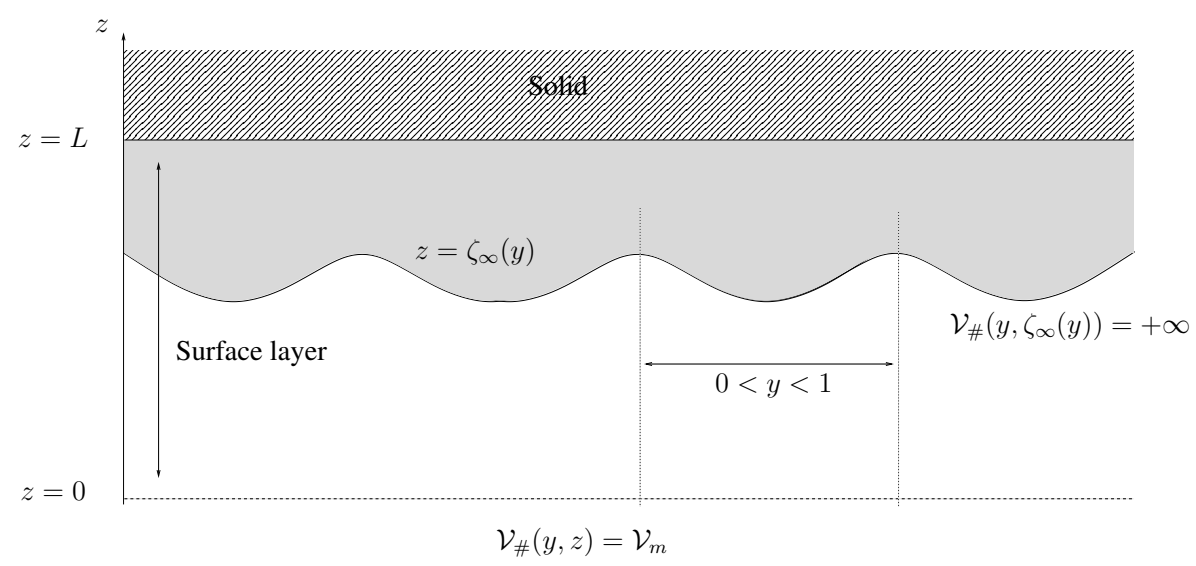

FIG. 1. Parametrization of the surface layer

of gas atoms is described by the following system of kinetic equations

$$
\begin{aligned}
& \partial_{t} f+\boldsymbol{v} \cdot \nabla_{x, z} f=Q_{m, m}[f, f], \quad z<0, \\
& \partial_{t} f+\boldsymbol{v} \cdot \nabla_{x, z} f-\frac{1}{m} \nabla_{x, z} \mathcal{V}(x, z) . \nabla_{\boldsymbol{v}} f=Q_{m, m}[f, f]+Q_{m, p}[f], \quad 0<z,
\end{aligned}
$$

where $Q_{m, m}$ is the Boltzmann collision operator (or a BGK like relaxation operator) and where the gas atom-phonon collision term reads (see for instance ${ }^{11}$ )

$$
\begin{array}{r}
Q_{m, p}[f]=\int K\left(E(x, z, \boldsymbol{v}), E\left(x, z, \boldsymbol{v}^{\prime}\right) ; T_{w}\right)\left(\mathcal{M}_{w}(E(x, z, \boldsymbol{v})) f\left(\boldsymbol{v}^{\prime}\right)\right. \\
\left.-\mathcal{M}_{w}(E(x, z, \boldsymbol{v})) f(\boldsymbol{v})\right) d \boldsymbol{v}^{\prime}
\end{array}
$$

with

$$
\mathcal{M}_{w}(E(x, z, \boldsymbol{v}))=\exp \left(-\frac{m|\boldsymbol{v}|^{2}}{2 k T_{w}}-\frac{\mathcal{V}(x, z)}{k T_{w}}\right)
$$


Nanoscale roughness effect on Maxwell-like boundary conditions

and $K\left(E, E^{\prime} ; T_{w}\right)$ is the probability of transition per unit time from the state $E^{\prime}$ to the state $E$ in a "collision" with a phonon. Moreover $Q_{m, p}$ satisfies the usual property of conservation of mass, an H-theorem and a symmetry property, and it has a Maxwellian equilibrium. Since we assume in all the following that the wall temperature $T_{w}$ is known and fixed, we will drop the dependence of $K$ on $T_{w}$.

Finally we introduce two times, $\tau_{m s}$ and $\tau_{f l}$, that will play a key role in the following and that are defined as follows: $\tau_{m s}$ is the time of relaxation of gas atoms by the phonons and is defined by

$$
\tau_{m s}=\tau_{m s}(E(x, z, \boldsymbol{v}))=\left(\int K\left(E, E^{\prime}\right) \mathcal{M}_{w}\left(E^{\prime}\right) d \boldsymbol{v}^{\prime}\right)^{-1}
$$

where $E^{\prime}=E\left(x, z, \boldsymbol{v}^{\prime}\right)$, while $\tau_{f l}(\boldsymbol{v})$ is the time of flight of a gas atom across the surface layer (without collision with a phonon): it depends on the incident velocity of the gas atom at the entrance of the surface layer, on the potential, and on the morphology of the wall.

\section{B. Derivation of boundary conditions for elastic scattering on a rough wall}

The derivation of boundary conditions for the Boltzmann equation on a wall with nanoscale roughness relies on a two scale asymptotic analysis from the above gassurface interaction model. In this section we only indicate the main ideas of this approach, skipping the technical mathematical details. The reader interested in a more rigorous presentation can refer to $\left(^{4}\right)$.

In many applications we have to consider rarefied flows on microtubes with a typical length of 1 to $10 \mathrm{~cm}$ (see for instance ${ }^{12}$ ). In such configurations a reasonable mesh size $l^{*}$ for a simulation based on the Boltzmann equation would be around $10 \mu \mathrm{m}$, so that $L^{*} \ll l^{*}$. Associated with those two space scales are two time scales $t_{B}^{*}$, the reference time of evolution of the Boltzmann equation defined by $t_{B}^{*}=l^{*} / v^{*}$ and $\tau_{f l}^{*}$, the reference time of flight of a gas atom across the surface layer defined 
Nanoscale roughness effect on Maxwell-like boundary conditions

by $\tau_{f l}^{*}=L^{*} / v^{*}$ where $v^{*}$ is a typical velocity of atoms of the gas flow (for instance $\left.v^{*}=\sqrt{k T_{w} / m}\right)$. Then we have $\tau_{f l}^{*} / t_{B}^{*}=L^{*} / l^{*} \ll 1$. In most applications we can also consider that the reference relaxation time $\tau_{m s}^{*}$ (that can be defined as $\left.\tau_{m s}\left(E\left(x, 0, v^{*}\right)\right)\right)$, is such that $\tau_{m s}^{*} \ll t_{B}^{*}$, but the interaction between the wall and the gas atoms depends mainly on the respective order of magnitude of the characteristic time scales $\tau_{f l}^{*}$ and $\tau_{m s}^{*}$. In this subsection we assume that $\tau_{f l}^{*} \ll \tau_{m s}^{*}$, that is to say that the time of flight of a gas atom across the surface layer is small enough so that the probability to have a collision with a phonon can be neglected. This is a limit regime but it can be relevant for low wall temperature or for light gas molecules.

Since the potential $\mathcal{V}$ is rapidly oscillating in the $x$-direction at the nanoscale, so is the solution of the system (4), at least near the surface layer. Thus a usual technique in the homogenization method is to look for a solution of (4) in the form (see ${ }^{13} 14$ )

$$
f(t, x, z, \boldsymbol{v})=F\left(t, x, x / L^{*}, z, \boldsymbol{v}\right)
$$

where the function $F(t, x, y, z, \boldsymbol{v})$ is periodic with respect to $y$ with period 1 . Note that with such a definition, we have

$$
\partial_{x} f=\partial_{x} F+\frac{1}{L^{*}} \partial_{y} F, \quad \partial_{x} \mathcal{V}=\partial_{x} \mathcal{V}_{\#}+\frac{1}{L^{*}} \partial_{y} \mathcal{V}_{\#}
$$

In the surface layer (for $z>0$ ), the variations of the function $F$ with respect to the variables $y$ and $z$ are significant while we can neglect the (much slower) variations of $F$ with respect to $t$ and $x$. And the right-hand side can also be neglected because we neglected the probability of collision between gas atoms and phonons (and even more the probability of collision between gas atoms). In the bulk flow we are interested in describing the variation of $F$ at the microscale (i.e. $l^{*}$ ) and the fast variation at the nanoscale $\left(L^{*}\right)$ can be taken into account only in average so that the bulk flow is described by the following distribution function

$$
g(t, x, z, \boldsymbol{v})=\int_{0}^{1} F(t, x, y, z, \boldsymbol{v}) d y, \quad \text { for } z<0
$$


Nanoscale roughness effect on Maxwell-like boundary conditions

Thus, in the limit regime we consider in this section, the gas flow can be described by the following simplified sytem

$$
\begin{gathered}
\partial_{t} g+v_{x} \partial_{x} g+v_{z} \partial_{z} g=Q_{m, m}[g, g], \quad z<0, \\
g\left(t, x, 0, v_{x}, v_{z}\right)_{v_{z}<0}=\int_{0}^{1} F\left(t, x, y, 0, v_{x}, v_{z}\right) d y \\
v_{x} \partial_{y} F+v_{z} \partial_{z} F-\frac{1}{2} \partial_{y} \mathcal{V}_{\#}(y, z) \partial_{v_{x}} F-\frac{1}{2} \partial_{z} \mathcal{V}_{\#}(y, z) \partial_{v_{z}} F=0, \quad 0<z, \\
F\left(t, x, y, 0, v_{x}, v_{z}\right)_{\mid v_{z}>0}=g\left(t, x, 0, v_{x}, v_{z}\right), \forall y \in[0,1] .
\end{gathered}
$$

This coupled system must be understood as follows. Equation (13) defines the distribution $F$ of gas atoms in a periodic cell of the surface layer: it depends on the distribution $\left.g\left(t, x, 0, v_{x}, v_{z}\right)\right|_{v_{z}>0}$ of gas atoms coming from the bulk flow that enter into the surface layer with $v_{z}>0$. Then the distribution $\left.F\left(t, x, 0, v_{x}, v_{z}\right)\right|_{v_{z}<0}$ of gas atoms that leave the surface layer (with $v_{z}<0$ ) can be, in principle, computed by solving (13)-(14). Finally, the distribution $g$ of gas atoms in the bulk flow is defined through (11)-(12). Relation (12) means that the distribution of gas atoms that come into the bulk flow from the surface layer is the average on one periodic cell of the distribution $\left.F\left(t, x, 0, v_{x}, v_{z}\right)\right|_{v_{z}<0}$ of gas atoms that leave the surface layer. In other words, relation (12) can be viewed as an implicit boundary condition for the bulk flow governed by (11), which requires to solve the boundary layer problem (13)-(14). This is summarized in Figure 2.

Now, we explain how a boundary condition for the bulk flow can be constructed. In fact, (13) can be written $\frac{d}{d s} F(t, x, y(s), z(s), \boldsymbol{v}(s))=0$ ( where $s \rightarrow(y(s), z(s), \boldsymbol{v}(s))$ is the trajectory of a gas atom across the surface layer, see equations (24)-(25) in section III). Then (13)-(14) can be integrated exactly, and the outgoing value $\left.F\left(t, x, y, 0, v_{x}, v_{z}\right)\right|_{v_{z}<0}$ can be written as a function of the incoming value (14). This outgoing value can be used in the implicit boundary condition (12), that can now be written explicitly as

$$
g(t, x, 0, \boldsymbol{v})_{\mid v_{z}<0}=\int_{0}^{1} g\left(t, x, 0,-\Lambda_{2}(y,-\boldsymbol{v})\right) d y,
$$

where $\Lambda\left(y^{\prime}, \boldsymbol{v}^{\prime}\right)=\left(y=\Lambda_{1}\left(y^{\prime}, \boldsymbol{v}^{\prime}\right), v=\Lambda_{2}\left(y^{\prime}, \boldsymbol{v}^{\prime}\right)\right)$ is the mapping that gives the position and velocity $(y, \boldsymbol{v})$ of a gas atom that leaves the surface layer (i.e. with 
Nanoscale roughness effect on Maxwell-like boundary conditions

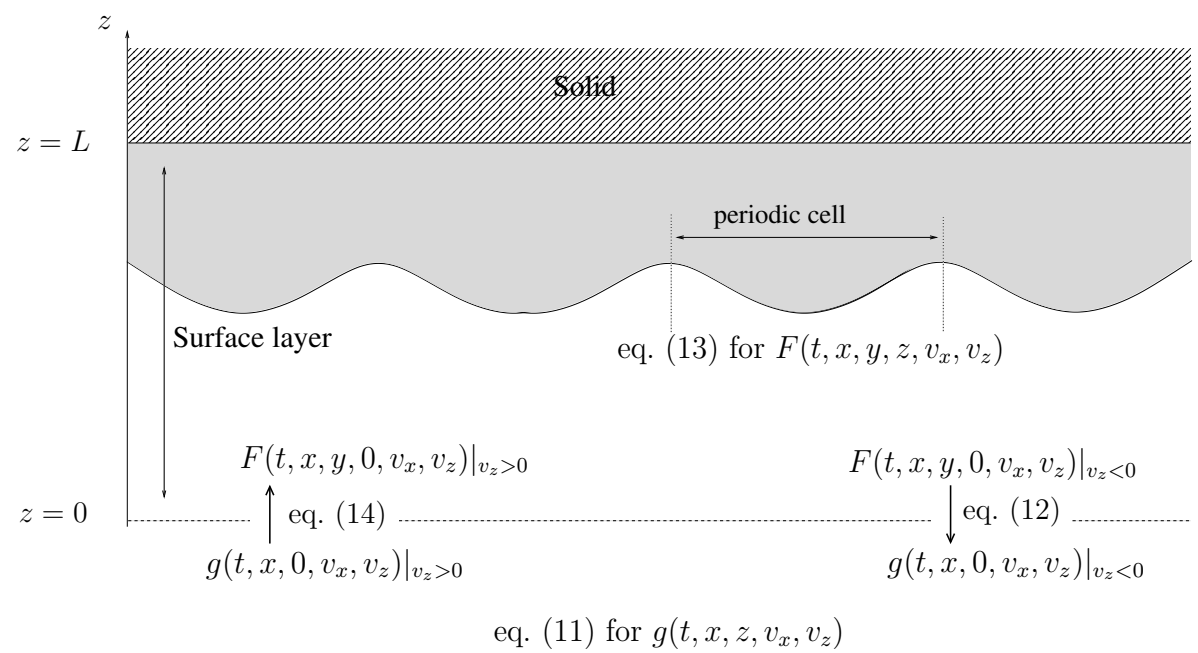

FIG. 2. Links between the bulk flow equation (11) and the boundary layer problem (13)(14)

$v_{z}<0$ at $\left.z=0\right)$ as a function of its position and velocity $\left(y^{\prime}, \boldsymbol{v}^{\prime}\right)$ when it enters into the surface layer (i.e. with $v_{z}^{\prime}>0$ at $z=0$ ), see figure 3. This mapping is defined through the equations of the trajectory of a gas atom (see section III and equations (24)-(25)).

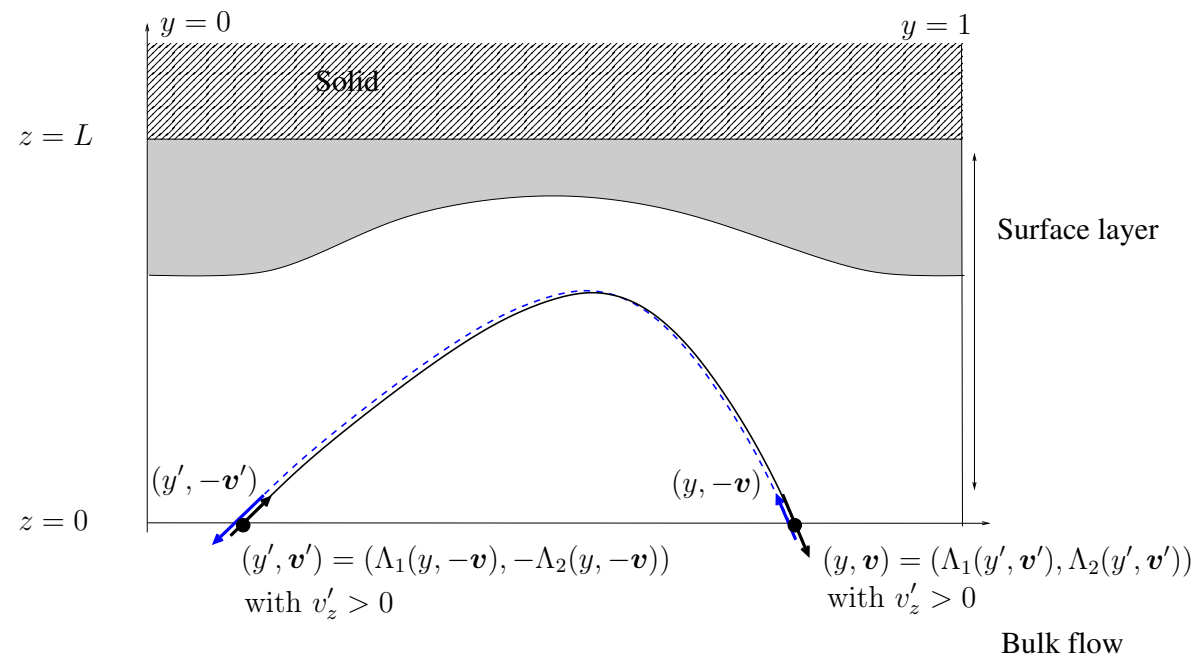

FIG. 3. One trajectory (and its corresponding reversed trajectory in blue) and the mapping $\Lambda$.

In other words, with a probabilistic interpretation, the incoming condition (14) 
means that a gas atom impinging the surface layer at point $(x, z=0)$ (at microscale), sees the nanoscale roughness from point $(x, y, z=0), y \in[0,1]$ with uniform probability in $y$. Equation (13) gives the deterministic trajectories of the gas atoms inside

the surface layer under the action of the interaction potential. Finally the outgoing distribution function at microscale is defined as the mean (in $y$ ) of the outgoing nanoscale distribution functions at point $(x, y, z=0)(14)$. After some algebra the reflection kernel of condition (15) is found to be

$$
g(t, x, 0, v)\left|v_{z}\right|_{\mid v_{z}<0}=\int_{v_{z}^{\prime}>0} R\left(\boldsymbol{v}^{\prime} \rightarrow \boldsymbol{v}\right) g\left(t, x, 0, \boldsymbol{v}^{\prime}\right)\left|v_{z}^{\prime}\right| d \boldsymbol{v}^{\prime},
$$

where

$$
R\left(\boldsymbol{v}^{\prime} \rightarrow \boldsymbol{v}\right)=\frac{\left|v_{z}\right|}{\left|v_{z}^{\prime}\right|} \int_{0}^{1} \delta\left(\boldsymbol{v}^{\prime}+\Lambda_{2}(y,-\boldsymbol{v})\right) d y
$$

and $\delta(y)$ is the Dirac delta function. This condition satisfies the properties of nonnegativeness, normalization and reciprocity and also a H-theorem, with an entropy production vanishing only for a flat wall ( $\operatorname{see}^{1}$ for a definition of these notions). Formula (17) clearly shows that the scattering kernel $R\left(\boldsymbol{v}^{\prime} \rightarrow \boldsymbol{v}\right)$ essentially depends on the trajectories of the gas atoms inside the surface layer (through the mapping $\Lambda$ ). See section III for an application of this reflection kernel with a given interaction potential. Note that it can be easily checked that, for a flat wall (i.e. for an interaction potential $\mathcal{V}(x, z)=\mathcal{V}(z)$ ), the boundary conditions (16) reduces to the classical specular reflection.

\section{Derivation of boundary conditions for "non-elastic" scattering on a rough wall}

We consider non-elastic scattering and in a first step we assume that

$$
\tau_{m s}^{*} \simeq \tau_{f l}^{*} \ll t_{B}^{*}
$$

This means that during their flight in the surface layer, some gas atoms have time to have a collision with a phonon. The same arguments as we used in the previous section lead to a system very similar to (11-14), except that equation (13) now 
Nanoscale roughness effect on Maxwell-like boundary conditions

contains a collision term in the right-hand side since gas atom-phonon collisions can occur:

$$
v_{x} \partial_{y} F+v_{z} \partial_{z} F-\frac{1}{2} \partial_{y} \mathcal{V}_{\#}(y, z) \partial_{v_{x}} F-\frac{1}{2} \partial_{z} \mathcal{V}_{\#}(y, z) \partial_{v_{z}} F=\frac{1}{\lambda_{*}} Q_{m, p}[F], \quad 0<z
$$

Again the left-hand side of (19) is nothing but the derivative of $F$ along the trajectories of gas atoms, and (19) can be written as

$$
\begin{aligned}
\frac{d}{d s} F(t, x, y(s), z(s), \boldsymbol{v}(s))= & \frac{1}{\lambda_{*}} Q_{m, p}^{+}[F(t, x, y(s), z(s), \boldsymbol{v}(s))] \\
& -\frac{F(t, x, y(s), z(s), \boldsymbol{v}(s))}{\tau_{m, s}(E)} .
\end{aligned}
$$

But because of the gain term $Q_{m, p}^{+}[F]$, it is not possible to get an explicit form for the solution of this equation. However, if we replace this term by $Q_{m, p}^{+}[\alpha(t, x) \mathcal{M}]$ where $\alpha(t, x)$ is a constant (with respect to the variables $y$ and $z$ ) to be defined later, it is easy to obtain the explicit solution (since $Q_{m, p}^{+}[\alpha(t, x) \mathcal{M}]$ and $\tau_{m s}(E)$ are constant along the characteristic):

$$
\begin{aligned}
F(t, x, y, 0, \boldsymbol{v})= & \exp (-r(y, \boldsymbol{v})) g\left(t, x, 0,-\Lambda_{2}(y,-\boldsymbol{v})\right) \\
& +(1-\exp (r(y, \boldsymbol{v}))) \alpha(t, x) \exp \left(-\mathcal{V}_{m}\right) M_{w}(\boldsymbol{v})
\end{aligned}
$$

where $M_{w}(\boldsymbol{v})=\exp \left(-m|\boldsymbol{v}|^{2} /\left(2 k T_{w}\right)\right.$ and $r(y, \boldsymbol{v})=\tau_{f l}(y,-\boldsymbol{v}) / \lambda_{*} \tau_{m s}(E(y, 0,-\boldsymbol{v}))$. With such a choice of the gain term we relax the (local) property of mass conservation of the collision term but we choose $\alpha$ so that the mass flux through the boundary vanishes (which is a global property of mass conservation) (see details in ${ }^{4}$ ). Finally, after some algebra we obtain the following boundary equation

$$
\begin{aligned}
g(t, x, 0, \boldsymbol{v})\left|v_{z}\right|_{\mid v_{z}<0}= & \int_{v_{z}^{\prime}>0} \overline{\mathcal{R}}\left(\boldsymbol{v}^{\prime} \rightarrow \boldsymbol{v}\right)\left|v_{z}^{\prime}\right| g\left(t, x, 0, \boldsymbol{v}^{\prime}\right) d \boldsymbol{v}^{\prime} \\
& +a(\boldsymbol{v}) \sigma\left[g\left(t, x, 0, \boldsymbol{v}^{\prime}\right)_{\mid v_{z}^{\prime}>0}\right]\left|v_{z}\right| M_{w}(\boldsymbol{v}),
\end{aligned}
$$

with

$$
\begin{aligned}
\overline{\mathcal{R}}\left(\boldsymbol{v}^{\prime} \rightarrow \boldsymbol{v}\right) & =\int_{0}^{1} \exp (-r(y, \boldsymbol{v})) \frac{\left|v_{z}\right|}{\left|v_{z}^{\prime}\right|} \delta\left(\boldsymbol{v}^{\prime}+\Lambda_{2}(y,-\boldsymbol{v})\right) d y \\
a(\boldsymbol{v}) & =1-\int_{0}^{1} \exp (-r(y, \boldsymbol{v})) d y \\
\sigma\left[g\left(t, x, 0, \boldsymbol{v}^{\prime}\right)_{\mid v_{z}^{\prime}>0}\right] & =\frac{\int_{v_{z}^{\prime}>0} v_{z}^{\prime}\left(1-\int_{0}^{1} \exp \left(-r\left(y^{\prime},-\boldsymbol{v}^{\prime}\right)\right) d y^{\prime}\right) g\left(t, x, 0, \boldsymbol{v}^{\prime}\right) d \boldsymbol{v}^{\prime}}{-\int_{v_{z}<0} v_{z}\left(1-\int_{0}^{1} \exp (-r(y, \boldsymbol{v})) d y\right) M_{w}(\boldsymbol{v}) d \boldsymbol{v}} .
\end{aligned}
$$


Note that the above coefficients have the following physical interpretations. First, $\exp (-r(y, \boldsymbol{v}))$ is the fraction of incident gas atoms re-emitted with velocity $\boldsymbol{v}$ which impinged the wall with velocity $\boldsymbol{v}^{\prime}=-\Lambda_{2}(y,-\boldsymbol{v})$ and that had no collision with phonons, and moreover this term does not depend on the approximation of the gain term. Then $\int_{0}^{1} \exp (-r(y, \boldsymbol{v})) d y=1-a(\boldsymbol{v})$ is the fraction of all the incident gas atoms re-emitted with velocity $\boldsymbol{v}$ (whatever incident velocity) and that had no collision with phonons. Finally, $a(\boldsymbol{v})$ is the fraction of incident gas atoms re-emitted with velocity $\boldsymbol{v}$ and that had a collision with phonons. Moreover, note that for a flat wall (i.e. if we assume that $\mathcal{V}(x, z)=\mathcal{V}(z)$ ), relation (22) yields

$$
g(t, x, 0, \boldsymbol{v})\left|v_{z}\right|_{\mid v_{z}<0}=(1-a(\boldsymbol{v}))\left|v_{z}\right| g(t, x, 0, \boldsymbol{v})+a(\boldsymbol{v}) \sigma(t, x)\left|v_{z}\right| M_{w}(\boldsymbol{v})
$$

where $a(\boldsymbol{v})$ is the fraction of incident gas atoms that have been thermalized. This relation is very similar to the classical Maxwell boundary condition but with a coefficient $a$ which depends-as in (22)-explicitly on the velocity $\boldsymbol{v}$ but also on the temperature $T_{w}$ (through $\tau_{m s}$, see $\left.(7)\right)$ and on the wall morphology (through $\tau_{f l}$ ).

The last regime to be studied is when we assume that $\tau_{m s}^{*} \ll \tau_{f l}^{*}$, that is to say that the relaxation time is much smaller than the time of flight which implies that all gas atoms entering the surface layer have a collision with a phonon and are thermalized. Then we get as it is expected the usual boundary condition of diffuse reflection (or total accommodation). This can be obtained either by an asymptotic analysis as in the previous regime studied above or by noting that

$$
\lim _{\tau_{m s} / \tau_{f l} \rightarrow 0} a(\boldsymbol{v})=1
$$

\section{IMPLEMENTATION OF MLBC IN A DVM CONTEXT}

In order to use the boundary conditions proposed in the previous sections, the potential energy surface $\mathcal{V}_{\#}$ has to be specified. Now we consider that the wall is a solid made up of atoms organized in a periodic structure which is assumed to be defect free. The potential $\mathcal{V}_{\#}$ is generated by the interaction potentials of these 
Nanoscale roughness effect on Maxwell-like boundary conditions

atoms $\left(\operatorname{see}^{19}\right)$, i.e. $\mathcal{V}_{\#}(y, z)=\sum_{i} \mathcal{V}_{i}(y, z)$ where each potential $\mathcal{V}_{i}$ is assumed to be a Lennard-Jones potential, with mixed-interaction parameters (in dimensionless form) $\tilde{\sigma}$ and $\tilde{\epsilon}$, centered at the position of an atom of the lattice

$$
\mathcal{V}_{i}(y, z)=V\left(r_{i}\right)=4 \tilde{\epsilon}\left(\left(\frac{\tilde{\sigma}}{\tilde{r}_{i}}\right)^{12}-\left(\frac{\tilde{\sigma}}{\tilde{r}_{i}}\right)^{6}\right)
$$

where $\tilde{r}_{i}=\sqrt{\left(y-y_{i}\right)^{2}+\left(\tilde{z}-\tilde{z}_{i}\right)^{2}}, \tilde{\epsilon}=2 \epsilon / k T$ and $\tilde{\sigma}=\sigma / L^{*}$. For the computations we will take only into account the atoms located in the periodic cell or in neighboring cells (see Fig. 4). With this definition of the potential energy surface, condition (2), which implies that the wall is non porous, is not satisfied, but this is not a problem for practical applications where the energy of incident gas atoms is not large enough to allow them to penetrate the wall. Note that in this configuration the roughness of the wall is of the order of the lattice period, i.e. at a very small scale (typically a nanometer or less). It is in the order of a standard experimental roughness for silicon channels or tubes of fused silica ${ }^{12} 1516$.

We first consider the simplest situation of elastic scattering where no atom is thermalized during the gas-surface interaction (see section II B). Then, as we indicated above, for a flat wall the boundary condition is the usual specular reflection. For a periodic rough wall the boundary condition introduced in ${ }^{4}$ is given by (16-17).

To make the boundary condition (16) explicit, we have to compute $\mathcal{R}\left(\boldsymbol{v}^{\prime} \rightarrow \boldsymbol{v}\right)$, or $\int_{0}^{1} \delta\left(v^{\prime}+\Lambda_{2}(y,-\boldsymbol{v})\right) d y\left(\right.$ for $v_{z}^{\prime}>0$ and $v_{z}<0$ ) which is the probability that an impinging gas atom with velocity $\boldsymbol{v}^{\prime}$ is re-emitted with velocity $\boldsymbol{v}$. At the same time, for a given $\boldsymbol{v}^{\prime}$, we obtain the scattering pattern of a mono-energetic beam of incident gas atoms by the wall and we can observe the effect of the roughness induced by our model. In the context of a numerical approximation of the Boltzmann (or BGK) equation by a discrete velocity method (DVM) ${ }^{17} 18$ we have to compute $\int_{0}^{1} \delta\left(\boldsymbol{v}_{p}^{\prime}+\Lambda_{2}\left(y,-\boldsymbol{v}_{q}\right)\right) d y$, for each pair $\left(\boldsymbol{v}_{p}^{\prime}, \boldsymbol{v}_{q}\right)$ in the set of discrete velocities. Since we are in a regime where the gas atom-phonon collisions are neglected (structure scattering $), \mathcal{R}\left(\boldsymbol{v}^{\prime} \rightarrow \boldsymbol{v}\right)$ is obtained by computing the trajectories of incident gas 


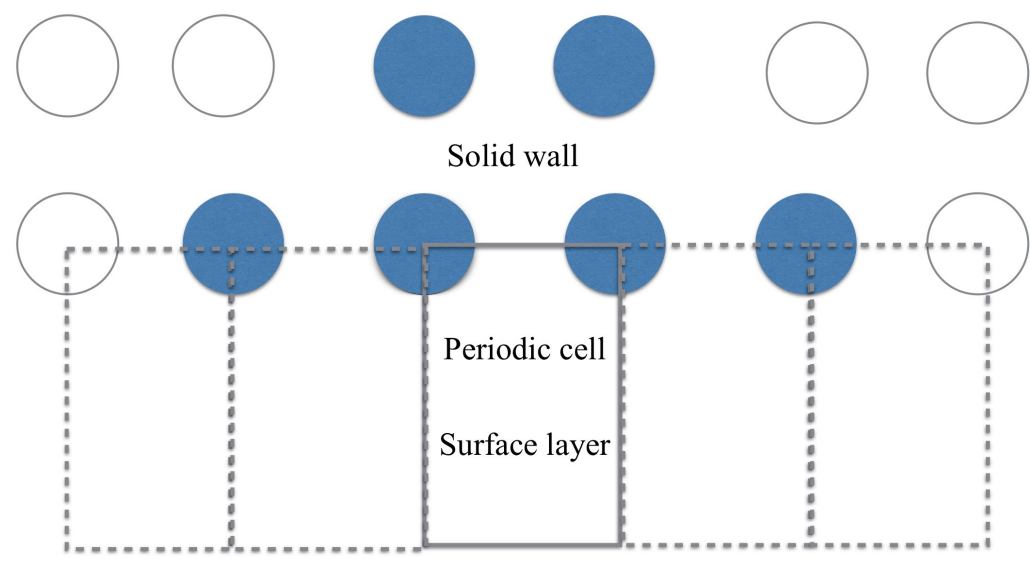

Bulk flow

FIG. 4. Periodic cell of the surface layer (2D case). The interaction potential of the colored atoms are taken into account

atoms with a given velocity $\boldsymbol{v}^{\prime}$ through the surface layer, which are the solutions of the system of ordinary differential equations describing the characteristic curves of $(13-14)$

$$
\begin{aligned}
& \dot{y}=v_{x}, \quad \dot{v}_{x}=-\partial_{y} \mathcal{V}(y, z), \\
& \dot{z}=v_{z}, \quad \dot{v}_{z}=-\partial_{z} \mathcal{V}(y, z) .
\end{aligned}
$$

It is a Hamiltonian system in which the total energy $E(y, z, \boldsymbol{v})=\frac{1}{2}\left(v_{x}^{2}+v_{z}^{2}\right)+\mathcal{V}(y, z)$ remains constant along the trajectories. It is numerically solved by using the velocity Verlet method which is a classical symplectic integrator. As a consequence of the energy conservation, the norm of the velocity of a gas atom leaving the surface layer is the same as when the gas atom entered the surface layer (since $\mathcal{V}(y, z=0$ ) is independent of $y$ ), but of course the re-emission angle has no simple relation with the incident angle. More precisely, if the velocity space is discretized by the following grid in polar coordinates:

$$
V=\left\{\boldsymbol{v}_{k, i}=r_{k}\left(\cos \theta_{i}, \sin \theta_{i}\right), 1 \leq k \leq k_{\max }, 1 \leq i \leq i_{\max }\right\}
$$


then $\mathcal{R}\left(\boldsymbol{v}_{k^{\prime}, i^{\prime}} \rightarrow \boldsymbol{v}_{k, i}\right)=0$ if $k \neq k^{\prime}$. On this grid, the distribution function is approximated by $f_{k, i} \approx f\left(\boldsymbol{v}_{k, i}\right)$. For a given incident velocity $\boldsymbol{v}^{i n c}=r_{i n c}\left(\cos \theta_{i n c}, \sin \theta_{\text {inc }}\right)$, several trajectories are computed for various equi-distributed initial condition $\mathrm{s}$ $\left(y_{m}, v_{y}^{i n c}, v_{z}^{i n c}\right)$, with $y_{m}=m \Delta y, 0 \leq m \leq m_{\max }, \Delta y=1 / m_{\max }$. For each trajectory we obtain the angle of reflection $\theta_{\text {ref }}$ of the gas atom in the flow $\left(\boldsymbol{v}^{\text {ref }}=\right.$ $\left.r_{\text {inc }}\left(\cos \theta_{\text {ref }}, \sin \theta_{\text {ref }}\right)\right)$. The interval $\left[-180^{\circ}, 0\right]$ of reflected angles is divided into $i_{\text {max }} / 2$ angular sectors (like the interval of incident angles). The $i$ th angular sector is $\left[-\theta_{i}-\pi /\left(2 i_{\max }\right),-\theta_{i}+\pi /\left(2 i_{\max }\right)\right]$, which is centered around $-\theta_{i}$ (where $\theta_{i}$ is the $i$ th discrete incident angle used to define the discrete velocity set). Boundary condition (16) is then discretized by

$$
\left|\sin \theta_{i}\right| f_{k, i}=\sum_{i^{\prime}=1}^{i_{\max }}\left|\sin \theta_{i^{\prime}}\right| \mathcal{R}\left(\boldsymbol{v}_{k^{\prime}, i^{\prime}} \rightarrow \boldsymbol{v}_{k, i}\right) f_{k, i^{\prime}} r_{k} \Delta r \Delta \theta,
$$

where $\Delta r=r_{k+1}-r_{k}$ and $\Delta \theta=\pi / i_{\max }$ are the (constant) steps of the velocity grid in the $r$ and $\theta$ directions. Note that in this relation, we used the fact that the terms in which $k \neq k^{\prime}$ are zero.

Note that for a practical use of this boundary condition, for a same wall material, the scattering kernel $\mathcal{R}\left(\boldsymbol{v}_{k^{\prime}, i^{\prime}} \rightarrow \boldsymbol{v}_{k, i}\right)$ can be computed and stored once for all.

\section{RESULTS AND DISCUSSION}

\section{A. Scattering pattern for elastic scattering on a rough wall}

As indicated above, when we compute $\mathcal{R}\left(\boldsymbol{v}^{\prime} \rightarrow \boldsymbol{v}\right)$ we obtain at the same time the scattering pattern of a mono-energetic beam of incident gas atoms by the wall and we can observe the effect of the roughness induced by our model. In our simulations the atoms of the wall are located in the periodic cell at points $(y=0, z=2),(y=1, z=2)$. The interaction potential of the wall is made up of the interaction potentials of these two atoms and of the adjacent atoms in the lattice (see Fig. 4). The temperature $T_{w}$ was set to $296 \mathrm{~K}, L^{*}$ to $0.4 \mathrm{~nm}$, the molecular mass $m=123 \mathrm{amu} \simeq 2.04 \times 10^{-25} \mathrm{~kg}$, so that the reference velocity is $v^{*}=200$ 
Nanoscale roughness effect on Maxwell-like boundary conditions

$\mathrm{m} / \mathrm{s}$ and the dimensionless parameters of the LJ potential are set to $\tilde{\sigma}=1, \tilde{\epsilon}=1$ (corresponding to $\sigma=0.4 \mathrm{~nm}$ and $\epsilon=2.0410^{-21} \mathrm{~J}$ ), and $L=2 \sigma=0.8 \mathrm{~nm}$.

In the following, we plot for each $i$ th angular sector the probability $p_{i}$ that an incident atom with velocity $\boldsymbol{v}^{i n c}$ is re-emitted in this sector. It is computed as follows: we count the number $n_{i}$ of gas atoms re-emitted in the $i$ th angular sector, and then we set $p_{i}=n_{i} / m_{\max }$, where $m_{\max }$ is the number of trajectories. The results presented below are computed with $m_{\max }=8001$ trajectories, since the results were not significantly modified when we used more trajectories. All trajectories were computed using a velocity Verlet method with a time step $\Delta t=2 \times 10^{-2}$ fs. For the visualization we take $i_{\max }=41$ so that each angular sector has an angle of $4.30^{\circ}$ and there is an average of 195.15 re-emitted atoms per angular sector.

\section{Influence of the angle of incidence}

In this simulation we take $\left|\boldsymbol{v}^{i n c}\right|=300 \mathrm{~m} / \mathrm{s}$, and $\theta^{i n c}$ takes the values (in degrees) $22.5^{\circ}, 45^{\circ}, 67.5^{\circ}, 90^{\circ}$.

Figure 5 shows very clearly that the scattering pattern is quite different from the usual specular reflection for any incident angle. For $\theta^{i n c}=90^{\circ}$ the diagram is symmetric and shows several peaks with a concentration of re-remitted particles near the specular reflection angle $\left(90^{\circ}\right)$. For the other values of $\theta^{i n c}$, the diagram shows a lobal distribution of backward scattered gas atoms with a peak more or less close to the specular reflection angle and a high but narrow peak of backward scattered gas atoms. More precisely, for $\theta^{i n c}=22.5^{\circ}$, the lobe shows several local peaks between $-45^{\circ}$ and $-75^{\circ}$ (for a specular angle of reflection equal to $-22,5^{\circ}$ ), and the peak of backward scattered gas atoms is around $-107^{\circ}$. For $\theta^{i n c}=45^{\circ}$, the lobe shows two local peaks around $-40^{\circ}$ and $-50^{\circ}$ for a specular reflection angle equal to $-45^{\circ}$ and the peak of backward scattered gas atoms is between $-121^{\circ}$ and $-125^{\circ}$. For $\theta^{i n c}=67.5^{\circ}$, the lobe shows a peak at $-70^{\circ}$, for a specular reflection angle equal to $67.5^{\circ}$ and the peak of backward scattered gas atoms is at $-147^{\circ}$. 
Nanoscale roughness effect on Maxwell-like boundary conditions

(a) $\theta^{\text {inc }}=22.5^{\circ}$

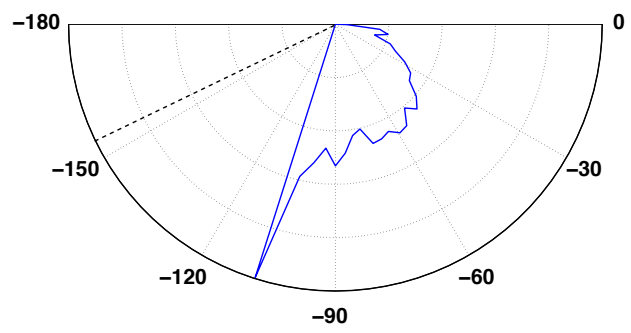

(c) $\theta^{\text {inc }}=67.5^{\circ}$

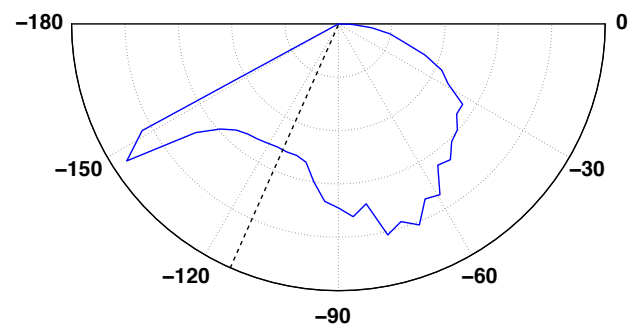

(b) $\theta^{\text {inc }}=45^{\circ}$

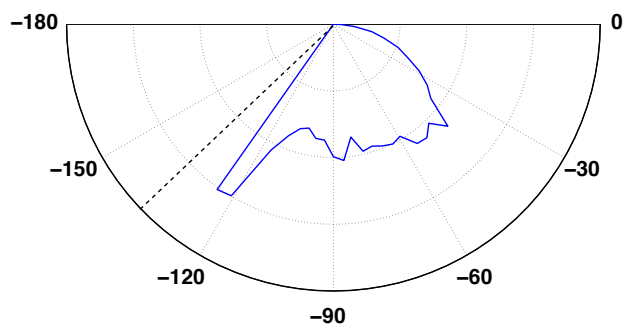

(d) $\theta^{\text {inc }}=90^{\circ}$

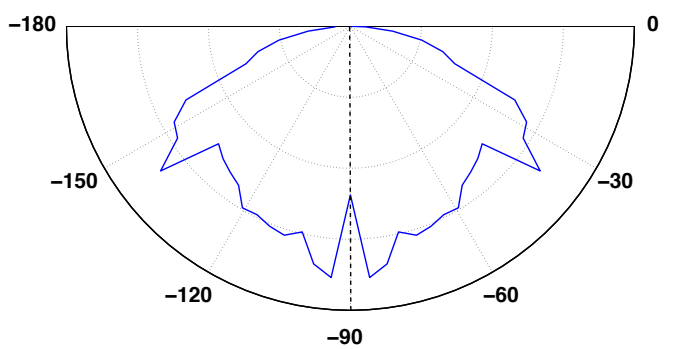

FIG. 5. Polar diagrams of the scattering pattern for impinging gas atoms with velocity $\left|\boldsymbol{v}^{i n c}\right|=300 \mathrm{~m} / \mathrm{s}$ and with incident angles $22.5^{\circ}$ (upper left), $45^{\circ}$ (upper right), $67.5^{\circ}$ (down left) and $90^{\circ}$ (down right). The incident and scattering angles are shown by dashed black line and the solid blue line respectively.

Influence of the energy (or mass) of the incident gas atoms

The trajectory of an incident gas atom through the surface layer depends on the position $(y, 0)$ where it enters the surface layer and on its incident kinetic energy (since $\mathcal{V}(y, 0)$ is independent of $y$ ). So changing the incident kinetic energy can be interpreted as keeping the same incident velocity but changing the mass of the gas atoms (i.e. changing the gas). The results given below are obtained by setting the angle of incidence to $45^{\circ}$ and setting the norm of the velocity to $\left|\boldsymbol{v}^{i n c}\right|=6 v^{*}=1200$ $\mathrm{m} / \mathrm{s}$. Figure 6 shows the computed polar diagrams of the scattering patterns for gas atoms with masses 123, 60, 16 and 2 amu. For heavy atoms ( $m=123 \mathrm{amu}$ ) we observe a small peak in the polar diagram for $\theta=-20^{\circ}$ and a larger one for $\theta=-85.6^{\circ}$. For $m=60 \mathrm{amu}$ the larger peak is for $\theta=-90^{\circ}$ and a smaller 
Nanoscale roughness effect on Maxwell-like boundary conditions

one for $\theta=-37.3^{\circ}$. The polar diagram corresponding to $m=16$ amu shows a backward scattering with a large peak at $\theta=-103.1^{\circ}$ and a forward scattering with a smaller peak at $\theta=-46^{\circ}$. Finally for the lightest atoms ( $\left.m=2 \mathrm{amu}\right)$ the diagrams shows also a backward scattering with a small peak at $\theta=-103.1^{\circ}$ and a forward scattering with larger peak at $\theta=-46^{\circ}$. Note that for light atoms $(m=16$ amu or $m=2 \mathrm{amu}$ ), the largest peak is obtained for forward scattered gas atoms with a reflection angle very close to the specular reflection angle $\theta=-45^{\circ}$. Finally we note that the scattering patterns depend significantly on the mass of the gas atom. This should be taken into account for simulating flows of mixtures of gas with different molecular masses, for instance when considering separation processes through micro-porous materials. Let us recall that the influence of the mass of the gas atom on the gas-surface interaction was shown by experiments in previous papers $^{12}{ }^{15}$. More precisely experiments suggest that the wall/gas interaction tends to be more diffuse with decreasing molecule mass and that the wall asperities can manage and scatter more easily the light incoming molecules. We note that the results of Fig. 6 are quite in agreement with those remarks .

\section{Influence of of the interaction potential}

The role of the parameters of the Lennard-Jones interaction potential is investigaded in Fig. 8. We compare the scattering pattern for an incident beam with $\theta^{i n c}=45^{\circ}$, $\left|v^{i n c}\right|=1200 \mathrm{~m} / \mathrm{s}$ with an interaction potential $V 1$ of parameters $\sigma=0.4 \mathrm{~nm}$ and $\epsilon=4.0810^{-21} \mathrm{~J}$ and for an interaction potential $V 2$ with parameters $\sigma=0.6 \mathrm{~nm}$ and $\epsilon=8.1610^{-22} \mathrm{~J}$ (see Fig. 7 (a)).

Both polar diagrams show two peaks but at very different angles: $-20^{\circ}$ and $-85^{\circ}$ for the first and $-35^{\circ}$ and $-55^{\circ}$ (closer to the specular reflection angle) for the other one. Those results indicates that the scattering pattern also strongly depends on the material of the wall. 
Nanoscale roughness effect on Maxwell-like boundary conditions

(a) $m=123 \mathrm{amu}$

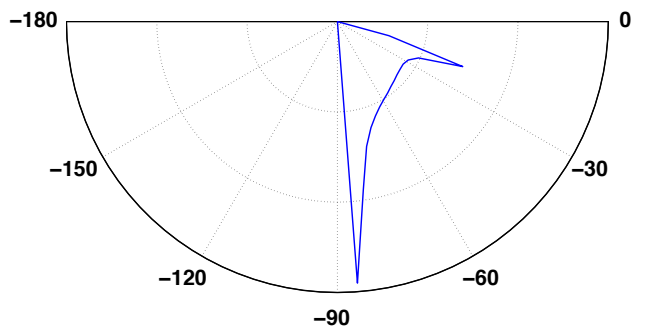

(c) $m=16 \mathrm{amu}$

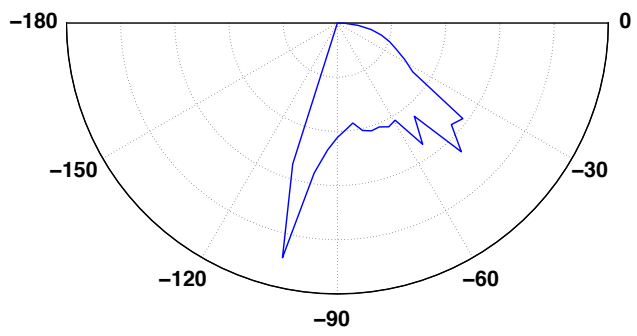

(b) $m=60 \mathrm{amu}$

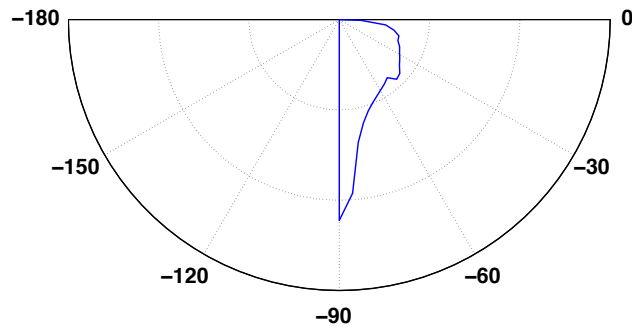

(d) $m=2 \mathrm{amu}$

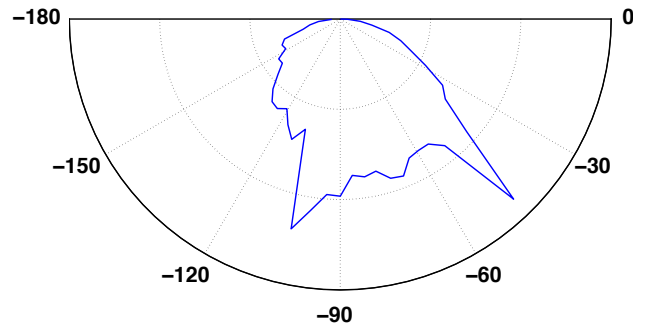

FIG. 6. Polar diagrams of the scattering pattern for impinging gas atoms with an incident angle $45^{\circ}$, a velocity $\left|\boldsymbol{v}^{i n c}\right|=1200 \mathrm{~m} / \mathrm{s}$ and for gas atom masses 123 (a), 60 (b), 16 (c), and 2 amu (d).

(a) Lennard-Jones potentials $V 1, V 2$

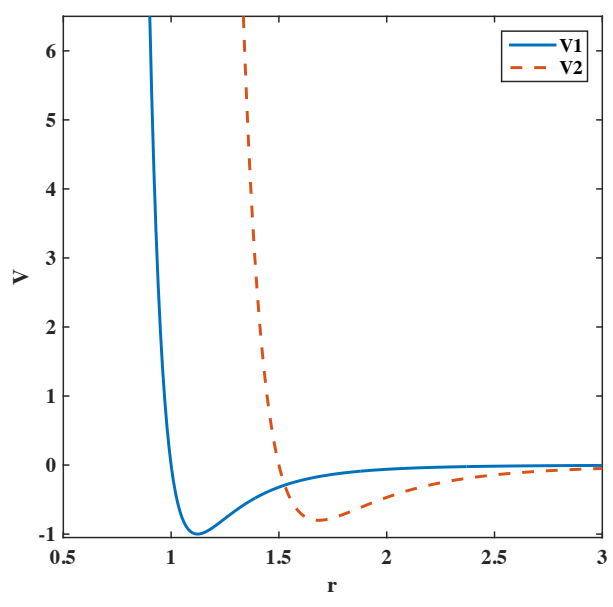

(b) lattice of wall atoms

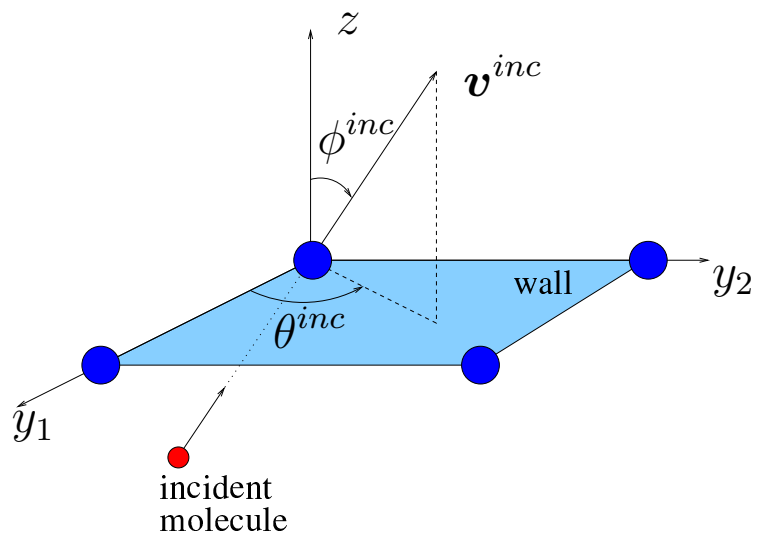

FIG. 7. Lennard-Jones potentials $V 1$ with $\sigma=0.4 \mathrm{~nm}$ and $\epsilon=2.0410^{-21} \mathrm{~J}$ and $V 2$ with $\sigma=0.6 \mathrm{~nm}$ and $\epsilon=1.6310^{-21} \mathrm{~J}$ (a) and lattice of wall atoms in 3D (b). 

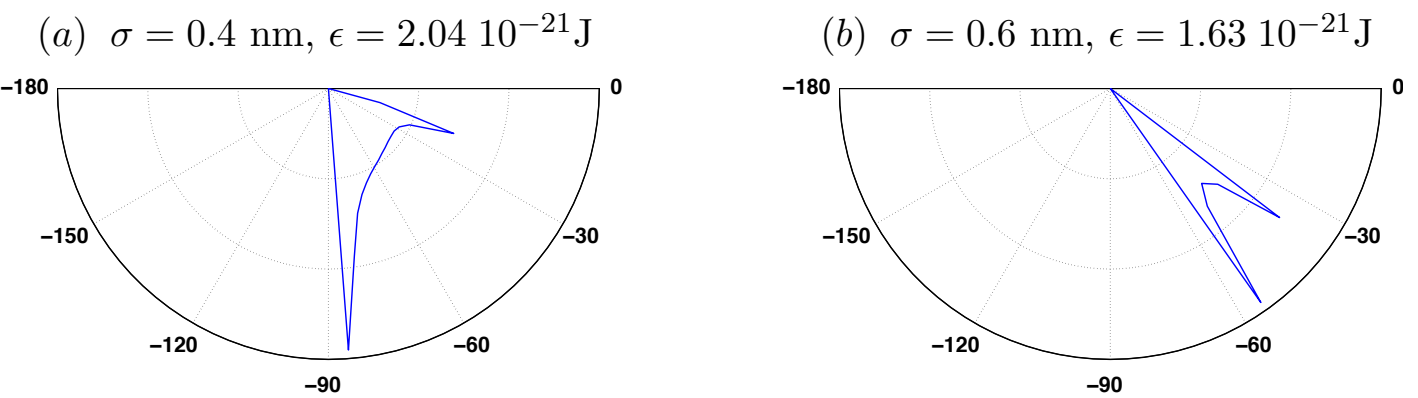

FIG. 8. Polar diagrams of the scattering pattern for impinging molecules with an incident angle $45^{\circ}$, a velocity $\left|\boldsymbol{v}^{i n c}\right|=1200 \mathrm{~m} / \mathrm{s}$, for a potential $V 1$ with $\sigma=0.4 \mathrm{~nm}$ and $\epsilon=$ $2.0410^{-21} \mathrm{~J}$ (a) and for a potential $V 2$ with $\sigma=0.6 \mathrm{~nm}$ and $\epsilon=1.6310^{-21} \mathrm{~J}(\mathrm{~b})$.

\section{Polar diagram of the scattering pattern in three dimensions}

The previous study can be extended to 3D. In this paragraph, scattering patterns are computed in a 3D configuration which is detailed below. The wall is described by a fcc lattice. The atoms in the periodic cell are located at points $\left(y_{1}=0, y_{2}=\right.$ $0, z=2),\left(y_{1}=1, y_{2}=0, z=2\right),\left(y_{1}=0, y_{2}=1, z=2\right),\left(y_{1}=1, y_{2}=1, z=2\right)$. The incident velocity is $\boldsymbol{v}^{i n c}=\left(v_{y_{1}}^{\text {inc }}, v_{y_{2}}^{\text {inc }}, v_{z}^{i n c}\right)$ with $v_{y_{1}}^{i n c}=\left|\boldsymbol{v}^{i n c}\right| \cos \theta^{i n c} \cos \phi^{i n c}, v_{y_{2}}^{\text {inc }}=$ $\left|\boldsymbol{v}^{i n c}\right| \sin \theta^{i n c} \cos \phi^{i n c}$ and $v_{z}=\left|\boldsymbol{v}^{i n c}\right| \sin \phi^{i n c}$, where $\phi^{i n c}$ is the incident angle with the surface and $\theta^{i n c}$ is the azimuthal angle (i.e. for $\theta^{i n c}=k \pi / 2$ the scattering plane is aligned with the lattice) (see Fig. $7(\mathrm{~b})$ ). The simulations were performed with $\left|\boldsymbol{v}^{\text {inc }}\right|=6000 \mathrm{~m} / \mathrm{s}, m=123 \mathrm{amu}, \phi^{i n c}=45^{\circ}$ and for two values of the azimuthal angle $\theta^{i n c}=90^{\circ}$, and $\theta^{i n c}=60^{\circ}$ and 10.000 equidistributed trajectories were computed.

On Fig. 8, the projections of the polar diagram of the scattering pattern on the plane $\left(v_{y_{1}}, v_{z}\right)$ and on the plane $\left(v_{y_{2}}, v_{z}\right)$ are shown for the two simulations with $\theta^{i n c}=90^{\circ}$ and $\theta^{i n c}=60^{\circ}$. For $\theta^{i n c}=90^{\circ}$, the scattering plane is aligned with the lattice while it is not aligned with the lattice for $\theta^{i n c}=60^{\circ}$. The influence of the azimuthal angle is noticeable. As expected the projection on the $\left(v_{y_{1}}, v_{z}\right)$ plane is symmetric when the scattering plane is aligned with respect to the symmetry axis of the surface lattice, while it is not at all symmetric when the scattering plane is misaligned $(\mathrm{d})$. Moreover the projections on the $\left(v_{y_{2}}, v_{z}\right)$ plane are quite different, 
Nanoscale roughness effect on Maxwell-like boundary conditions
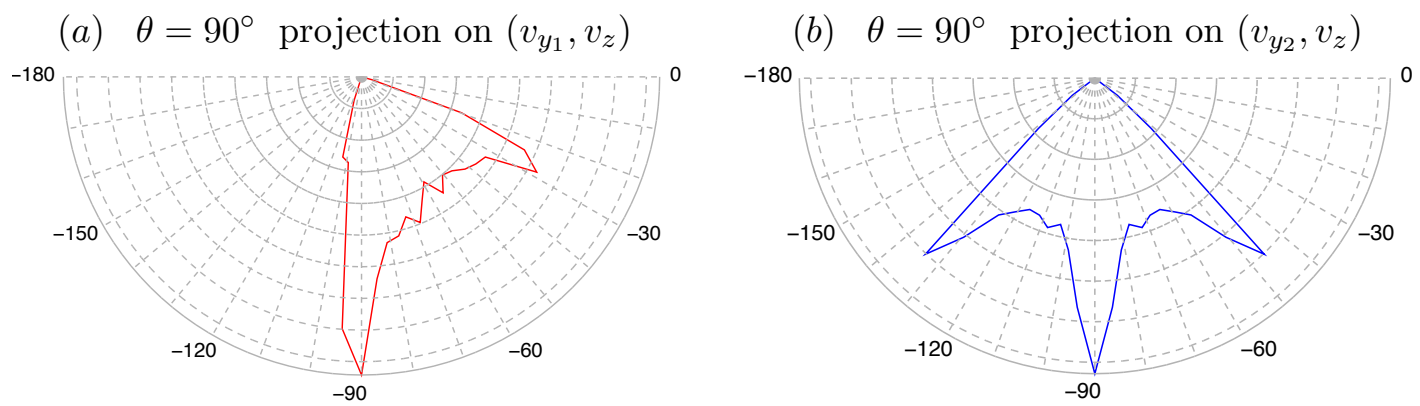

(c) $\theta=60^{\circ}$ projection on $\left(v_{y_{1}}, v_{z}\right)$

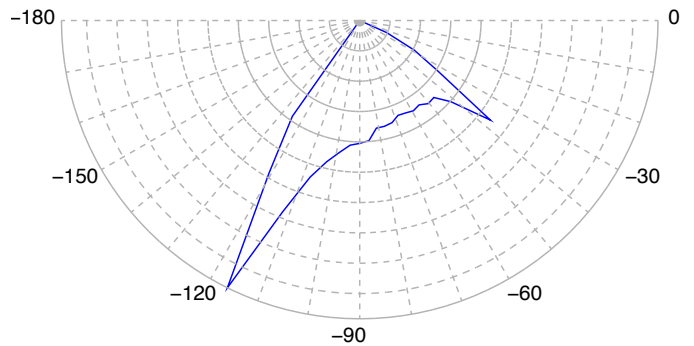

(d) $\theta=60^{\circ}$ projection on $\left(v_{y_{2}}, v_{z}\right)$

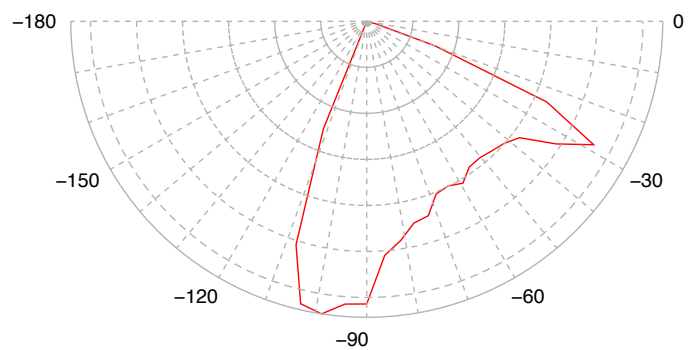

FIG. 9. Projection of the polar diagram of the scattering pattern on $\left(v_{y_{1}}, v_{z}\right)$ for $\theta=90^{\circ}$ (a), on $\left(v_{y_{2}}, v_{z}\right)$ for $\theta=90^{\circ}(\mathrm{b})$, on $\left(v_{y_{1}}, v_{z}\right)$ for $\theta=60^{\circ}(\mathrm{c})$, and on $\left(v_{y_{2}}, v_{z}\right)$ for $\theta=60^{\circ}$ (d).

showing a large peak of backward scattered gas atoms (around $-98^{\circ}$ ) for $\theta^{i n c}=60^{\circ}$.

\section{B. Non-elastic scattering on a rough wall}

As shown in IIC, the fraction of gas atoms that are thermalized during the interaction with the surface is given in our model by

$$
a(\boldsymbol{v})=1-\int_{0}^{1} \exp \left(-\tau_{f l}(y,-\boldsymbol{v}) /\left(\lambda_{*} \tau_{m s}(E(y, 0,-\boldsymbol{v}))\right) d y\right.
$$

and so depends on $\boldsymbol{v}, \tilde{\epsilon}$, and $\tilde{\sigma}$, through $\tau_{f l}$, and also on $T_{w}$, the temperature of the wall, through $\tau_{m s}$. The time of flight $\tau_{f l}$ is easily obtained when computing the trajectory of the gas molecules in the surface layer by solving (24-25). The time $\tau_{m s}$ is a decreasing function of the wall temperature $T_{w}$, but is unfortunately more difficult to evaluate. Until now we lack experiments, but approximations could be 
Nanoscale roughness effect on Maxwell-like boundary conditions

obtained through molecular dynamics simulations. This is a difficult problem by itself and will be treated in forthcoming publications. Here, we follow a simplified approach $^{9}$ that consists in approximating $a$ by

$$
a(\boldsymbol{v})=1-\int_{0}^{1} \exp \left(-\tau_{f l}(y,-\boldsymbol{v}) /\left(\lambda_{*} \tau_{m s}^{\text {mean }}\left(T_{w}\right)\right) d y,\right.
$$

where $\tau_{m s}^{\text {mean }}\left(T_{w}\right)$ is an average relaxation time due to gas atom-phonon collisions. In order to investigate the effects of the temperature of the solid, we simulate $a(\boldsymbol{v})$ as a function of $\tau_{m s}^{\text {mean }}$. Since $\tau_{m s}^{\text {mean }} \geq 10^{-13} \mathrm{~s}$ (the characteristic period of the thermal vibration of atoms in a solid ${ }^{9}$ ), and takes values roughly in the range $10^{-13}-10^{-12} \mathrm{~s}$ $\left(\sec ^{91120}\right.$ ), we studied the variation of $a(\boldsymbol{v})$ for $0.2510^{-12} \mathrm{~s} \leq \tau_{m s}^{\text {mean }} \leq 1.410^{-11} \mathrm{~s}$ (Fig. 10a), for a beam of impinging gas atoms with $m=123 \mathrm{amu}, \theta^{i n c}=45^{\circ}$ and $\lambda_{*}=2$. We use a simple numerical approximation of the integral of (26) to get:

$$
a(\boldsymbol{v}) \approx 1-\sum_{m=1}^{m_{\max }} \exp \left(-\tau_{f l}\left(y_{m},-\boldsymbol{v}\right) /\left(\lambda_{*} \tau_{m s}^{\text {mean }}\left(T_{w}\right)\right) \Delta y,\right.
$$

where $\tau_{f l}\left(y_{m},-\boldsymbol{v}\right)$ is the time for a particle that enters into the surface layer in $y_{m}=m \Delta y$ with a velocity $-\boldsymbol{v}$ to leave this surface: it was computed exactly when trajectories equations (24-25) were solved in section IV A.

As expected, the fraction of incident gas atoms that have been thermalized decreases. For $\tau_{m s}^{\text {mean }}=0.2510^{-12} \mathrm{~s}$, the fraction is very closed to 1 , which means that we are in the regime of total accommodation, while for $\tau_{m s}^{\text {mean }}=1.410^{-11} \mathrm{~s}$ this fraction is very small so that the scattering pattern is mainly governed by the morphology of the wall ("structure scattering"). Figure 10b shows the dependence of the fraction $a(\boldsymbol{v})$ on the the velocity. We simulate $\left|\boldsymbol{v}^{i n c}\right|$ from $200 \mathrm{~m} / \mathrm{s}$ up to 10000 $\mathrm{m} / \mathrm{s}$ for $\tau_{m s}^{\text {mean }}=0.52510^{-12} \mathrm{~s}$ and $m=123 \mathrm{amu}$. It appears that for small velocities, the coefficient $a \approx 0.8$ while for $\left|\boldsymbol{v}^{i n c}\right|=2400 \mathrm{~m} / \mathrm{s} a \approx 0.13$, and we are close to an elastic scattering, which is a consequence of the smaller times of flight $\tau_{f l}(y)$ of each trajectory.

Finally the influence of the incident angle on the coefficient $a$ is shown in Table I where the simulations were performed with $\lambda_{*}=2$ and $\tau_{m s}^{\text {mean }}=0.52510^{-12} \mathrm{~s}$. It is 
Nanoscale roughness effect on Maxwell-like boundary conditions

(a) $a(\boldsymbol{v})$ versus relaxation time

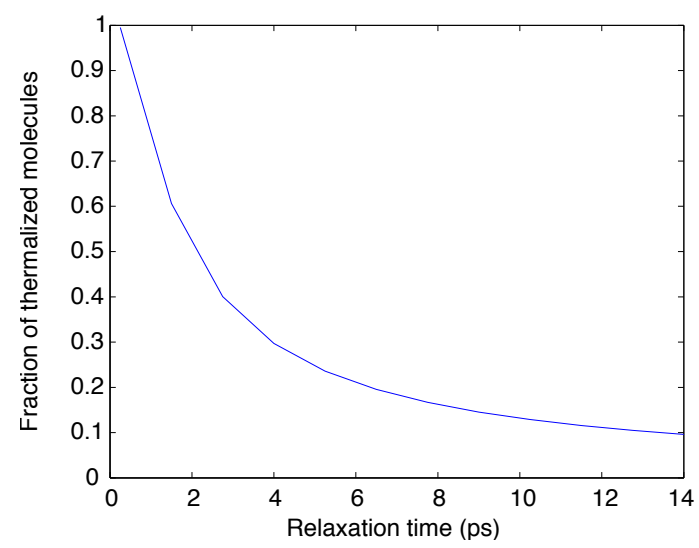

(b) $a(\boldsymbol{v})$ versus incident velocity

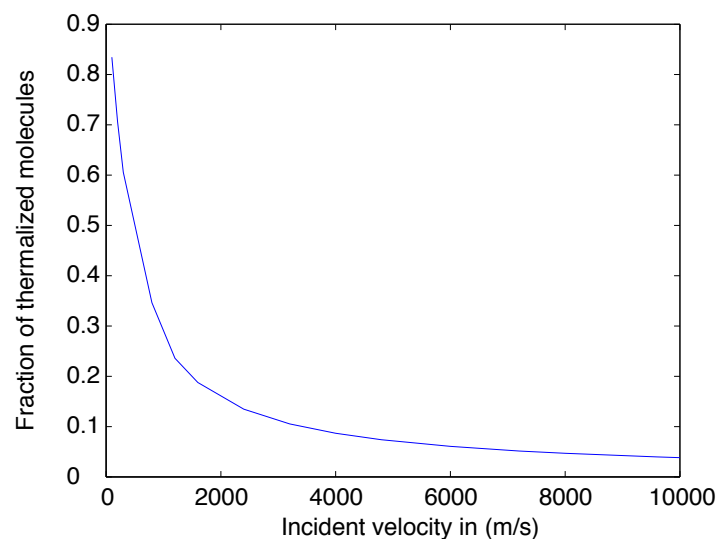

FIG. 10. Fraction $a(\boldsymbol{v})$ of thermalized molecules, for (a) $\left|\boldsymbol{v}^{i n c}\right|=1200 \mathrm{~m} / \mathrm{s}, \theta^{\text {inc }}=45^{\circ}$, $\lambda_{*}=2$, as a function of $\tau_{m s}^{\text {mean }}$, and for (b) $\theta^{i n c}=45^{\circ}, \tau_{m s}^{\text {mean }}=5.2510^{-12} \mathrm{~s}, \lambda_{*}=2$, as a function of $\left|\boldsymbol{v}^{i n c}\right|$.

TABLE I. Fraction of thermalized gas atoms as a function of the incident angle

\begin{tabular}{cc}
\hline \hline incident angle. & fraction of thermalized gas atoms. \\
\hline $22.5^{\circ}$ & 0.3736 \\
$45^{\circ}$ & 0.23 \\
$67.5^{\circ}$ & 0.1999 \\
$90^{\circ}$ & 0.1906 \\
\hline
\end{tabular}

clear that the smaller the incident angle, the larger the fraction of thermalized gas atoms, as a consequence of larger times of flight.

\section{CONCLUSION}

We have proposed new boundary conditions allowing to take into account at the kinetic level the effect of the van der Waals forces and of the periodic roughness of the wall at the atomic scale. This model relies on a two scale theoretical approach whose mathematical framework has been introduced in a previous paper. We have proposed a way to include some physics of gas-surface interaction by computing the 
Nanoscale roughness effect on Maxwell-like boundary conditions

trajectories of the impinging gas atoms through a periodic cell of the surface layer in which gas molecules interact with surface atoms by a simplified PES. Then it is possible to get an approximation of the scattering kernel of the new boundary condition suitable for a DVM numerical approach of the Boltzmann equation. We have shown the scattering pattern of a mono-energetic beam of impinging gas atoms reflected by a wall with nanoscale roughness. Several simulations on a simplified 2D configuration or on a $3 \mathrm{D}$ configuration prove that the model brings at the kinetic scale information on the roughness of the wall at the atomic scale which are not present in usual boundary conditions. 2D simulations of elastic scattering show that the scattering pattern is strongly dependent on several parameters such as the incident angle of the impinging gas atoms, their energy or the mixed-interaction parameters of the Lennard-Jones potential. Moreover 3D computations prove the influence of the azimuthal angle of the scattering plane. For non-elastic scattering a simplified form of the new boundary condition shows that the temperature of the wall and the incident angle of the impinging gas atoms have a noticeable influence on the fraction of thermalized gas atoms. But further investigations are necessary to obtain a more precise evaluation of the relaxation time of gas atoms by the phonons. This needs some more experiments and/or numerical Molecular Dynamics simulations and will be treated in forthcoming publications. All those simulations are promising and prove that our approach is simple enough to be numerically tractable, while it is able to include enough physics of gas-surface interaction so as to improve kinetic simulations of micro or nano-flows in narrow channels, and hence could be a useful tool in this context.

\section{REFERENCES}

${ }^{1}$ C. Cercignani, The Boltzmann Equation and its Applications, (Applied Mathematical Sciences 67, Springer Verlag, 1988).

${ }^{2}$ H. Struchtrup, "Maxwell boundary condition and velocity dependent accommodation coefficient", Phys. Fluids 25, 112001 (2013). 
Nanoscale roughness effect on Maxwell-like boundary conditions

${ }^{3}$ M. H. Gorji and P. Jenny, "A gas-surface interaction kernel for diatomic rarefied gas flows based on the Cercignani-Lampis-Lord model", Phys. Fluids 26, 122004 (2014).

${ }^{4}$ S. Brull, P. Charrier and L. Mieussens, "Gas-surface interaction and boundary conditions for the Boltzman equation", Kinetic and Related Models 7, No 2 (2014).

${ }^{5}$ R. M. Logan and R. E. Stickney, "Simple Classical Model for the Scattering of Gas Atoms from a Solid Surface", J. Chem. Phys. 44, 195 (1966).

${ }^{6}$ J. C. Tully, "Washboard model of gas-surface scattering, J. Chem. Phys. 92, 680 (1990).

${ }^{7}$ J. C. Tully, "Dynamics of gas-surface interactions: 3D generalized Langevin model applied to fcc and bcc surfaces", J. Chem. Phys. 73, 975 (1980).

${ }^{8}$ P. Gamallo, L. Martin-Gondre, R. Sayós, C. Crespos, P. Larrégaray , "Potential Energy Surfaces for the Dynamics of Elementary Gas-Surface Processes", Dynamics of Gas-Surface Interactions, Springer Series in Surface Sciences 50, 25 (2013).

${ }^{9}$ V. D. Borman, S. Yu. Krylov and A. V. Prosyanov, "Theory of nonequilibrium phenomena at a gas-solid interface", Sov. Phys. JETP 67, 2110 (1988).

${ }^{10}$ K. Aoki, P. Charrier and P. Degond, "A hierarchy of models related to nanoflows and surface diffusion", Kinetic and Related Models 4, No 1, 53 (2011).

${ }^{11}$ S. Yu Krylov, A. V. Prosyanov and J. J. Beenakker, "One dimensionnal surface diffusion. II Density dependence in a corrugated potential", J. Chem. Phys. 107, 6970 (1997).

${ }^{12}$ P. Perrier, I. A. Graur, T. Ewart and J. G. Méolans, "Mass flow rate measurements in microtubes: from hydrodynamic to near free molecular regime", Phys. Fluids 23, 042004 (2011).

${ }^{13}$ E. Skjetne, J. L. Auriault, "Homogenization of wall-slip gas flow ithrough porous media, Transp. Porous Med. 36, 293 (1999).

${ }^{14}$ E. Sanchez-Palencia, Non homogeneous media and vibration theory, (Lecture Notes in Physics, 127, Springer 1980).

${ }^{15}$ I. A. Graur, P. Perrier, W. Ghozlani and J. G. Molans, "Measurement of tangential momentum accomodation coefficient for various gases in plane microchannel", 
Nanoscale roughness effect on Maxwell-like boundary conditions

Phys. Fluids 21, 102004 (2009).

${ }^{16}$ T. Gronych, R. Ulman, L. Peksa, P. Repa, "Measurements of the relative momentum accomodation coefficient for different gases with a viscosity vacuum gauge", Vacuum 73, 275 (2004).

${ }^{17}$ F. Rogier and J. Schneider, "A direct method for solving the Boltzmann equation", Transport Theory and Statistical Physics 23, 313 (1994).

${ }^{18}$ L. Mieussens, "Discrete-Velocity Models and Numerical Schemes for the Boltzmann-BGK Equation in Plane and Axisymmetric Geometries", J. Comput. Phys. 162, 429-466 (2000).

${ }^{19}$ S. Brull and P. Charrier, "Boundary conditions for the Boltzmann equation for rough walls",in Proceedings of the 29th International Symposium on Rarefied Gas Dynamics, Xi'an, China, 13-18 July 2014, edited by Jing Fan,, AIP Conf. Proc. 1628, 368 .

${ }^{20}$ J. J. Beenaker, Y. Krylov, "One dimensionnal surface diffusion: Density dependence in a smooth potential, J. Chem. Phys. 107, 4015 (1997). 